\title{
Endometrial intraepithelial neoplasia with secretory differentiation: diagnostic features and underlying mechanisms
}

\author{
Carlos E Parra-Herran, Nicolas M Monte and George L Mutter \\ Department of Pathology, Women's and Perinatal Pathology Division, Harvard Medical School, Brigham and \\ Women's Hospital, Boston, MA, USA
}

\begin{abstract}
Endometrial intraepithelial neoplasia (EIN) with secretory differentiation and ordinary EIN occurring in a secretory context are rare but recognized findings. We determined how often secretory differentiation in EIN was associated with evidence of circulating progestins in the background endometrium, and studied clinical characteristics and clinical outcomes of affected patients. We selected 41 patients with secretory differentiation in either the EIN itself $(n=31)$ and/or background endometrium $(n=38)$. Most $(90 \%, 28 / 31)$ secretory EINs were associated with circulating progestins. Rare exceptions were observed, suggesting that secretory EIN may occur as a hormone-independent phenomenon. Circulating progestins are not sufficient, however, to induce EIN secretory differentiation, as $26 \%(10 / 38)$ of EIN within a secretory background were of the ordinary (nonsecretory) type. EIN patients with secretory endometrium in the background are younger (averaging 45 years) than the aggregate group of all patients with EIN (53 years in previously published studies) and are often premenopausal with a cyclical source of endogenous progestins. Involution of EIN during follow-up was more frequent $(81 \%, 17 / 21)$ for those with a secretory background at the time of initial EIN diagnosis compared with historical averages $(25 \%, 36 / 142)$. These results suggest a potential role for endogenous progesterone, as well as therapeutic progestins, in modulating EIN outcomes.
\end{abstract}

Modern Pathology (2013) 26, 868-873; doi:10.1038/modpathol.2012.231; published online 18 January 2013

Keywords: endometrial cancer; endometrial intraepithelial neoplasia; secretory

Endometrial intraepithelial neoplasia (EIN) is a clonal proliferation of endometrial glands, considered to be a premalignant condition due to its strong association with concurrent and/or subsequent endometrioid (type I) adenocarcinoma of the endometrium. Indeed, a diagnosis of EIN is associated with a $27 \%$ likelihood of having ('concurrent') adenocarcinoma within 1 year, and carrying a 45fold increased risk for a future (after 1 year) diagnosis of adenocarcinoma. ${ }^{1}$ Diagnosis of EIN requires strict application of histological criteria, including size (at least $1 \mathrm{~mm}$ in greatest linear dimension), architecture (area of glands exceeds area of endometrial stroma) and cytology (difference in nuclear and cytoplasmic appearance between the abnormal and background endometrium). ${ }^{2-4}$

Correspondence: Dr Professor GL Mutter, MD, Department of Pathology, Women's and Perinatal Pathology Division, Harvard Medical School, Brigham and Women's Hospital, 75 Francis Street, Boston, MA 02115, USA.

E-mail: gmutter@rics.bwh.harvard.edu

Received 10 October 2012; revised 24 November 2012; accepted 6 December 2012; published online 18 January 2013
Exclusion of cancer, as well as benign conditions mimicking EIN, is an important part of accurate diagnosis.

Accurate and sensitive diagnosis of EIN in reproductive age women can be challenging when a secretory background is present. Secretory-phase endometrium displays changes that may morphologically overlap with EIN. ${ }^{2}$ In normal mid-late secretory endometrium the gland to stroma ratio is increased, nuclei become enlarged and round, and large expanses of secretory superficial endometrium are offset from basal areas lacking secretory changes. Furthermore, when EIN does occur within the context of a secretory environment, its presence may be masked by relative crowding of the flanking normal glands, and appearance of secretory vacuoles within the EIN itself.

Neoplastic glands of EIN can display different types of differentiation, including squamous, tubal, mucinous and secretory phenotypes. Secretory differentiation has been reported in approximately $5 \%$ of sequential cases of EIN. ${ }^{5}$ Although infrequent, EIN with secretory differentiation may be deceptively bland. Progestin therapy of EIN is 
often accompanied by nuclear shrinkage in neoplastic glands, a paradoxical change given that the opposite (nuclear enlargement) occurs in normal progestin exposed glands. ${ }^{6}$ This can mask the cytological atypia expected in atypical endometrial hyperplasia, but usually remains evident as a relative cytological change in direct comparison of areas of EIN with background. Therefore, secretory change in endometrium, both neoplastic and nonneoplastic, is a potential diagnostic confounder.

Although the presence of secretory change in EIN has been reported, a careful analysis of its hormonal setting and implications for the histopathological diagnosis of EIN has not been systematically explored. Similarly, only a few studies of 'endometrial hyperplasia' have analyzed the presence of secretory change. The aim of this study is to analyze the presence of secretory differentiation in cases of EIN and correlate it with demographic data, hormonal status, histopathological features of neoplastic and background endometrium, and clinical outcome.

\section{Materials and methods}

This study was reviewed and approved by the BWH Committee for the Protection of Human Subjects. We screened 191 sequential pathology reports for specimens received between May 2005 and March 2011, in which the pathology report mentioned secretory EIN $(n=70)$, or EIN within a secretory background $(n=121)$. In all, 174 cases were excluded (109 consults from other institutions with no material available, 13 cases from our institution with no material available, 9 cases with concurrent cancer, 10 cases reported as subdiagnostic of EIN, 3 cases representing subsequent samples from patients already included in our sample). Slides were retrieved from the remaining 47 cases, and reviewed by two pathologists to confirm the presence of both EIN and background endometrium in the slides, as well as secretory change in either the EIN or background. Two cases were excluded because they contained no residual EIN lesion on the slides available, and four cases were excluded because we could not confirm the presence of any secretory change. This left a total of 41 unique patient specimens with EIN plus background endometrium and secretory changes in the specimen. The specimen used for entry into our study was usually an endometrial biopsy or curettage $(61 \%, 25 / 41)$, with the remaining being hysterectomy specimens.

The 41 accepted specimens were first independently scored by two pathologists (GLM and CPH) for a variety of specific histological features, including type of secretory changes present within the EIN, normal background endometrium and stromal progestin-related changes (predecidua). These individual histological features, several of which might be present in one case, were collected independently of each reviewing pathologists, and analyzed in aggregate. Following this, a review by both pathologists together was performed for an overall consensus classification of each of the 41 cases as yes/no secretory changes in EIN and yes/no progestin effect in the background endometrium (secretory glands and/or stromal decidualization).

Clinical annotation was retrieved by medical record review (patients were not contacted), including patient demographic and clinical outcome data. The later included type of therapy instituted after diagnosis of EIN (hormonal vs surgical treatment) and the rate of remission (no EIN or cancer on subsequent biopsy, curetting or hysterectomy), persistence (EIN in subsequent biopsy, curetting or hysterectomy) and disease progression (endometrial endometrioid adenocarcinoma in subsequent biopsy, curetting or hysterectomy).

\section{Results}

Table 1 shows distribution of all 41 accepted cases of EIN ('all EIN') according to the consensus determination of the presence of secretory change within the EIN itself $(n=31)$ compared with histological evidence of circulating progestins as indicated by secretory glands and/or decidualized stroma in the background endometrium $(n=38)$.

Figure 1 illustrates combinations of secretory differentiation between EIN and background endometrial compartments. Of the 38 EINs seen in a secretory background endometrium, most $(74 \%, 28$ / 38) (Figures 1a and b), but not all (Figures 1c and d), showed secretory differentiation within the EIN itself. Less common was secretory differentiation within EIN glands in those patients lacking evidence of circulating progestins (normal endometrium was inactive or atrophic in these cases) $(n=3$; Figures 1 e and f).

Overall, $76 \%$ of all cases (31/41) upon consensus review showed secretory change within the EIN, based on the presence of specific cytological features, with some cases having multiple features. EIN glandular changes indicative of secretory

Table 1 Distribution of secretory change in neoplastic (EIN, either 'ordinary' or 'secretory') and non-neoplastic (background, with or without progesterone effect) glandular endometrium

Secretory change in EIN, n (\%)

\begin{tabular}{lccc}
$\begin{array}{l}\text { Secretory } \\
\text { Background }\end{array}$ & Absent & Present & Total, n (\%) \\
\hline $\begin{array}{l}\text { Absent } \\
\text { Present }\end{array}$ & $10(24 \%)$ & $3(7.3 \%)$ & $3(7.3 \%)$ \\
Total & $10(24 \%)$ & $31(76 \%)$ & $41(100 \%)$ \\
\hline
\end{tabular}

EIN $=$ endometrial intraepithelial neoplasia.

${ }^{a}$ Not eligible for study. See Materials and methods. 

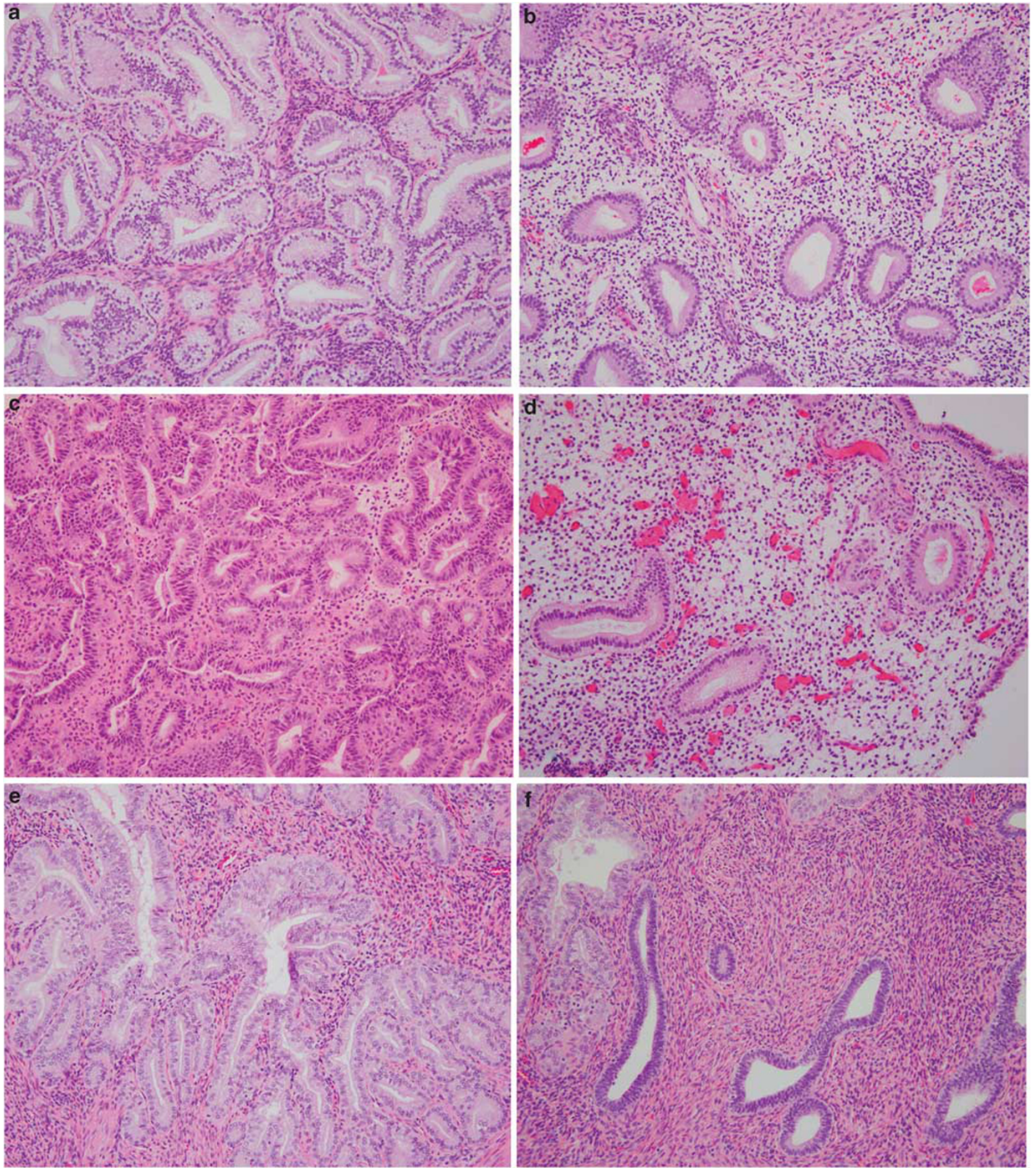

Figure 1 Patterns of secretory change in endometrial intraepithelial neoplasia (EIN) (a, c and e) and corresponding normal background endometrium (b, d and f). (a and b) Secretory EIN in a background of secretory endometrium. In this case, secretory changes (including supra- and subnuclear vacuolization) are more pronounced in neoplastic glands (hematoxylin and eosin (H\&E), $\times 200)$. (c and d) Conventional (non-secretory) EIN in a background of early secretory endometrium in a pre-menopausal woman. In this case, circulating progestins do not produce secretory differentiation in neoplastic glands $(\mathrm{H} \& \mathrm{E}, \times 200)$. (e and f) Secretory EIN in a background of inactive endometrium in a post-menopausal woman. Secretory changes in neoplastic glands include nuclear rounding and cytoplasmic expansion with ruffled apical borders. Each row is one patient $(\mathrm{H} \& \mathrm{E}, \times 200)$.

differentiation included supra- and/or infranuclear vacuoles $(31 \%)$, clearing of the cytoplasm $(38 \%)$ and presence of a ruffled apical epithelial border (40\%).
Each subgroup from Table 1 was separately analyzed. Demographic and clinical data are displayed in Table 2. Average age at the time of 
Table 2 Demographic data, clinical presentation and relevant gynecological history

\begin{tabular}{|c|c|c|c|}
\hline & $E I N+$ prog & $s E I N+$ prog & sEIN no prog \\
\hline & $\begin{array}{c}\text { Ordinary EIN with progestin } \\
\text { background, } \mathrm{N}=10\end{array}$ & $\begin{array}{c}\text { Secretory EIN with progestin } \\
\text { background, } \mathrm{N}=28\end{array}$ & $\begin{array}{l}\text { Secretory EIN, without progestin- } \\
\text { altered background, } \mathrm{N}=3\end{array}$ \\
\hline Age (years) & $44.8(8.3,23.6-53.1)$ & $27.3(7.8,27.3-58.5)$ & $59.8(\mathrm{NA}, 51.9-70.3)^{\mathrm{a}}$ \\
\hline Body mass index & $25.9(6.1,20.2-38.5)$ & $31.4(7.0,22.3-42.8)$ & $27.8(\mathrm{NA}, 24-31.5)^{\mathrm{a}}$ \\
\hline Gravity & $1.6(1.3,0-3)$ & $1.7(2.1,0-10)$ & $2(\mathrm{NA}, 1-3)^{\mathrm{a}}$ \\
\hline Parity & $1.3(1.3,0-3)$ & $1.2(1.2,0-4)$ & $2(\mathrm{NA}, 1-3)^{\mathrm{a}}$ \\
\hline Post-menopause & $0 \%(0 / 10)$ & $17 \%(5 / 28)$ & $67 \%(2 / 3)$ \\
\hline History of irregular menstrual periods & $70 \%(7 / 10)$ & $69 \%(18 / 26)$ & $33 \%(1 / 3)$ \\
\hline History of oligomenorrhea & $10 \%(1 / 10)$ & $0 \%(0 / 26)$ & $0 \%(0 / 3)$ \\
\hline History of post-menopausal bleeding & $0 \%(0 / 10)$ & $18 \%(5 / 28)$ & $33 \%(1 / 3)$ \\
\hline History of PCOS & $13 \%(1 / 8)$ & $20 \%(5 / 25)$ & $33 \%(1 / 3)$ \\
\hline Current HRT & $0 \%(0 / 10)$ & $11 \%(3 / 28)$ & $0 \%(0 / 3)$ \\
\hline Past HRT & $0 \%(0 / 8)$ & $8 \%(2 / 26)$ & $33 \%(1 / 3)$ \\
\hline Current IUD use & $0 \%(0 / 9)$ & $0 \%(0 / 27)$ & $0 \%(0 / 3)$ \\
\hline Past IUD use & $0 \%(0 / 7)$ & $6 \%(1 / 17)$ & $0 \%(0 / 3)$ \\
\hline Current OCP use & $22 \%(2 / 9)$ & $3.7 \%(1 / 27)$ & $0 \%(0 / 3)$ \\
\hline Past OCP use & $67 \%(4 / 6)$ & $50 \%(5 / 10)$ & $100 \%(1 / 1)$ \\
\hline Current Progestin use & $33 \%(3 / 9)$ & $36 \%(10 / 28)$ & $0 \%(0 / 3)$ \\
\hline Past progestin use & $71 \%(5 / 7)$ & $57 \%(13 / 23)$ & $100 \%(3 / 3)$ \\
\hline Current Tamoxifen use & $22 \%(2 / 9)$ & $0 \%(0 / 27)$ & $67 \%(2 / 3)$ \\
\hline Past Tamoxifen use & $22 \%(2 / 9)$ & $0 \%(0 / 27)$ & $67 \%(2 / 3)$ \\
\hline Surgical treatment (hysterectomy) & $40 \%(4 / 10)$ & $39 \%(11 / 28)$ & $33 \%(1 / 3)$ \\
\hline
\end{tabular}

$\mathrm{PCOS}=$ polycystic ovarian syndrome; HRT = hormone replacement therapy; IUD = intrauterine device, OCP = oral contraceptive; $\mathrm{NA}=$ not applicable.

${ }^{\mathrm{a}}$ Units are: mean (standard deviation, range).

diagnosis in the entire study group (averaged 46 years overall for 41 patients) was significantly younger $(P<0.01)$ than the literature reported average age of patients with any EIN of 53 years. ${ }^{7}$ This difference is largely contributed by the young age of two largest patient groups (EIN + prog and sEIN + prog), having evidence of circulating progestins in the background endometrium and only $13 \% \quad(5 / 38)$ of whom were clinically postmenopausal. The most frequent presenting symptoms were irregular menstrual periods or postmenopausal bleeding.

Many of our patients were taking one or more type of hormonal supplements at the time of the initial sample in which EIN was diagnosed (Table 2). The most common was a progestin-containing therapy, found in approximately a third of cases overall. Less than half of the cases with histological evidence of circulating progestins in the background endometrium (groups EIN + prog and sEIN + prog) had a history of any current hormone use (infertility treatments, oral contraceptives, hormonal replacement therapy or Tamoxifen). In all other cases, the source of progestins responsible for the secretory changes is, therefore, most likely endogenous.

Short-term clinical outcomes are shown in Table 3 for each of the subgroups. By combining the first two subgroups, we can summarize outcomes for all women with EIN (whether sEIN or ordinary EIN) having evidence of circulating progestins at the time of initial EIN diagnosis. This is a total of 21 patients (EIN + prog and sEIN + prog groups) with average follow-up of 134 days. Nineteen percent (4/21) received hormonal treatment after diagnosis of EIN, not significantly different $(P=0.844)$ from the historical average at our institution previously reported for sequentially presenting EIN cases $(17 \%, 23 / 133) .{ }^{7}$ Sixty-six percent $(14 / 21)$ finally underwent hysterectomy with the balance monitored by repeat sampling. Interestingly, histological regression of EIN to a benign histology was observed in the majority, $76 \%$ of cases $(16 / 21)$, with EIN persistence $(19 \%, 4 / 21)$ and progression to endometrial adenocarcinoma $(5 \%, 1 / 21)$ much less common. This regression rate of $76 \%$ for EIN presenting with evidence of circulating progestins was significantly higher $(P<0.001)$ than that seen in our historical comparison group of all EIN cases $(25 \%, 36 / 142)$.

\section{Discussion}

Irrespective of its endogenous or exogenous origin, circulating systemic progestins are capable of initiating secretory differentiation within EIN lesions. Much more rarely, secretory EIN occurs independently as part of the neoplastic phenotype. Using the changes in background endometrium as an indicator of circulating progesterone, the majority of secretory EINs (93\%) occurred in patients with evidence of circulating progestins. Most were premenopausal, and thus some proportion might be attributed to endogenous progesterone production 
Table 3 Management and clinical outcome of patients with EIN and secretory changes diagnosed on biopsy/curettage

\begin{tabular}{|c|c|c|c|c|}
\hline & $E I N+$ prog & $s E I N+$ prog & sEIN no prog & $\begin{array}{c}\text { Historical } \\
\text { comparison } \\
\text { series (7) }\end{array}$ \\
\hline & $\begin{array}{l}\text { Ordinary EIN with } \\
\text { progestin background }\end{array}$ & $\begin{array}{l}\text { Secretory EIN with } \\
\text { progestin background }\end{array}$ & $\begin{array}{c}\text { Secretory EIN, without } \\
\text { progestin-altered background }\end{array}$ & $\begin{array}{l}\text { Sequential } \\
\text { EIN cases }\end{array}$ \\
\hline \multicolumn{5}{|l|}{ Follow-up interval } \\
\hline Total follow-up available, $N$ & 6 & 15 & 2 & 142 \\
\hline Average (median, range) in days & $134(70,21-356)$ & $209(128,22-681)$ & 27 (NA, 7-47) & $269(74, \mathrm{NA})$ \\
\hline \multicolumn{5}{|l|}{ Medical $R x$} \\
\hline Progestin therapy for EIN & $33 \%$ & $13 \%$ & $50 \%$ & $17 \%$ \\
\hline \multicolumn{5}{|l|}{ Final specimen type } \\
\hline Biopsy & $0 \%$ & $13 \%$ & $0 \%$ & \\
\hline Curettage & $33 \%$ & $20 \%$ & $0 \%$ & \\
\hline Hysterectomy & $67 \%$ & $67 \%$ & $100 \%$ & \\
\hline \multicolumn{5}{|c|}{ Histological diagnosis in final specimen } \\
\hline Benign & $83 \%$ & $73 \%$ & $50 \%$ & $25 \%$ \\
\hline EIN & $17 \%$ & $20 \%$ & $50 \%$ & $35 \%$ \\
\hline Cancer & $0 \%$ & $6.6 \%$ & $0 \%$ & $39 \%$ \\
\hline
\end{tabular}

$\mathrm{NA}=$ not applicable or not available

secondary to the physiological menstrual cycle. This matches the observations of other investigators, who have reported a premenopausal age of most patients with endometrial hyperplasia with secretory change. ${ }^{8}$ Careful review of the medical record discloses, however, that $35 \%(13 / 37)$ of our patients with evidence of circulating progestins had already been administered pharmacological exogenous progestins at the time of endometrial sampling, presumably for empirical symptomatic relief of irregular or abnormal bleeding.

The use of exogenous progestins is an increasingly documented, if non-standardized, therapeutic option in women with EIN that are young or have contraindications for surgical management. Immunohistochemistry for estrogen and progesterone receptor show high levels within EIN glands, indicating their potential ability to respond to these hormones. ${ }^{6}$ Response to oral progestin hormonal therapy is seen in about $77 \%$ of cases with a longterm complete response of $53 \%$, being more prevalent in cases of atypical endometrial hyperplasia than in cases of adenocarcinoma. ${ }^{9,10}$ More recently, local delivery by progestinimpregnated intrauterine device has also shown to be effective in some patients, even with successful pregnancies thereafter. ${ }^{11}$ Glands with molecular alterations such as PTEN inactivation appear to be particularly sensitive to exogenous progestins, as PTEN-null glands preferentially disappear after treatment in most cases. ${ }^{12}$

In cases of EIN with a secretory background at presentation, the presence of endogenous circulating progesterone may have an antiproliferative effect in neoplastic glands, similar to that seen with exogenous progestin therapies. This 'endogenous therapy' may contribute to the relatively high rate $(76 \%)$ of EIN regression in such cases compared with what we have seen previously $(25 \%)$ for all patients with EIN at our hospital. The difference cannot be attributed to a higher rate of progestin therapy following EIN diagnosis, as that was similar for both.

The diagnosis of EIN can be challenging in the presence of secretory changes, whether within the EIN or background. This is due to a combination of changes in the lesion and background that may perturb application of diagnostic criteria. First, progestins inhibit glandular proliferation and induce apoptosis, resulting in decreased glandular cellularity and nuclear changes, including reduction in nuclear size and loss of 'atypia'.2,6,13,14 Despite these absolute changes that may occur over the course of time with therapy, contrast between lesion and background cytology is generally maintained throughout and thus more informative than fixed definitions of cytological atypia. We made the diagnosis of EIN only when the cytology of crowded glands exceeding $1 \mathrm{~mm}$ in size, although secretory, differed from the cytological changes found in the background. Perhaps, even more difficult are those stromal changes that occur in response to circulating progestins. Within EIN lesions, the stromal compartment may expand with decidualized stroma thus reducing gland density, and thereby reducing sensitivity of EIN detection. Correspondingly, normal secretory glands late in the menstrual cycle can develop redundant secretory epithelium within gland tracts that may be closely apposed, at quite high density. Coordinated changes in cytology and architecture must be demonstrated to maintain specificity of EIN diagnosis in a 
secretory background. However, when strict criteria for the diagnosis of EIN are applied, as in our study, this risk is minimized. The well-documented reproducibility of EIN in clinical practice ${ }^{15}$ and the high concordance between the two reviewers in this study make the possibility of misdiagnosis unlikely.

Limitations of our study include relatively short follow-up of a modest number of patients, and lack of a formally matched secretory-free comparison group. Instead, we used a previously published comparison group of sequential EINs from the same institution, which, of note, lacked pre-selection for secretory phenotype within the EIN or background. Progesterone levels were not measured in any of our patients at the time of diagnosis. We believe, however, that the absence of background secretory changes is good evidence of lack of significant effects of this hormone on the endometrium.

In summary, secretory differentiation within EIN is found predominantly in premenopausal women, and such differentiation usually reflects the presence of circulating progesterone, as indicated by the histology of the background endometrium. Irrespective of secretory change within the EIN itself, endometrial evidence of circulating progestins at the time of initial EIN diagnosis confers a tendency towards EIN regression greater than that seen for EIN cases overall. The precise magnitude of this effect, and the best way to measure it in the individual patient, is insufficiently defined at present to apply this parameter to clinical management of patients presenting with EIN. Further studies of larger numbers of patients with longer clinical follow-up will be valuable in determining the clinical significance of endogenous circulating progestins in the natural history of EIN.

\section{Disclosure/conflict of interest}

The authors declare no conflict of interest.

\section{References}

1 Baak JP, Mutter GL, Robboy S, et al. The molecular genetics and morphometry-based endometrial intraepithelial neoplasia classification system predicts disease progression in endometrial hyperplasia more accurately than the 1994 World Health Organization classification system. Cancer 2005; 103:2304-2312.
2 Mutter GL, Zaino RJ, Baak JPA, et al. Benign endometrial hyperplasia and EIN, In: Robboy SJ, Mutter GL, Prat J, Bentley R, Russell P, Anderson MC(eds). Robboy's Pathology of the Female Reproductive Tract, 2nd edn. Elsevier: New York, NY; 2009, pp 367-391.

3 Mutter GL, Zaino RJ, Baak JPA, et al. The benign endometrial hyperplasia sequence and endometrial intraepithelial neoplasia. Int J Gynecol Pathol 2007; 26:103-114.

4 Silverberg SG, Mutter GL, Kurman RJ, et al. Tumors of the uterine corpus: epithelial tumors and related lesions, In: Tavassoli FA, Stratton MR (eds). WHO Classification of Tumors: Pathology and Genetics of Tumors of the Breast and Female Genital Organs, 1st edn. IARC Press: Lyon; 2003, pp 221-232.

5 Carlson JW, Mutter GL. Endometrial intraepithelial neoplasia is associated with polyps and frequently has metaplastic change. Histopathology 2008;53:325-332.

6 Lin MC, Lomo L, Baak JPA, et al. Squamous morules are functionally inert elements of premalignant endometrial neoplasia. Mod Pathol 2009;22:167-174.

7 Semere LG, Ko E, Johnson NR, et al. Endometrial intraepithelial neoplasia clinical correlates and outcomes: a practice-based experience. Obstet Gynecol 2011;118:21-28.

8 Tresserra F, Lopez-Yarto M, Grases PJ, et al. Endometrial hyperplasia with secretory changes. Gynecol Oncol 2003;88:386-393.

9 Gunderson CC, Fader AN, Carson KA, et al. Oncologic and reproductive outcomes with progestin therapy in women with endometrial hyperplasia and grade 1 adenocarcinoma: a systematic review. Gynecol Oncol 2012;125:477-482.

10 Randall TC, Kurman RJ. Progestin treatment of atypical hyperplasia and well-differentiated carcinoma of the endometrium in women under age 40. Obstet Gynecol Surv 1997;90:434-440.

11 Minig L, Franchi D, Boveri S, et al. Progestin intrauterine device and $\mathrm{GnRH}$ analogue for uterussparing treatment of endometrial precancers and welldifferentiated early endometrial carcinoma in young women. Ann Oncol 2011;22:643-649.

12 Zheng W, Baker HE, Mutter GL. Involution of PTENnull endometrial glands with progestin therapy. Gynecol Oncol 2004;92:1008-1013.

13 Wheeler DT, Bristow RE, Kurman RJ. Histologic alterations in endometrial hyperplasia and well-differentiated carcinoma treated with progestins. Am J Surg Pathol 2007;31:988-998.

14 Amezcua CA, Lu JJ, Felix JC, et al. Apoptosis may be an early event of progestin therapy for endometrial hyperplasia. Gynecol Oncol 2000;79:169-176.

15 Usubutun A, Mutter GL, Saglam A, et al. Reproducibility of endometrial intraepithelial neoplasia diagnosis is good, but influenced by the diagnostic style of pathologists. Mod Pathol 2012;25:877-884. 